\title{
Doppler Detection of Extrasolar Planets
}

\author{
G. W. Marcy ${ }^{1}$
}

Dept. of Physics and Astronomy, San Francisco State Univ., San Francisco, CA 94132

\section{R. Paul Butler}

Anglo-Australian Observatory, NSW 2121, Epping, Australia

\section{A. Fischer}

Dept. of Physics and Astronomy, San Francisco State Univ., San Francisco, CA 94132

\begin{abstract}
.
We have measured the radial velocities of $540 \mathrm{G}$ and $\mathrm{K}$ main sequence stars with a precision of $3-10 \mathrm{~ms}^{-1}$ using the Lick and Keck échelle spectrometers. We had detected 6 companions that have $m \sin i$ $<7 M_{\text {Jup }}$. We announce here the discovery of a new planet around Gliese 876, found in our Doppler measurements from both Lick and Keck. This is the first planet found around an M dwarf, which indicates that planets occur around low-mass stars, in addition to solar-type stars. We combine our entire stellar sample with that of Mayor et al. to derive general properties of giant planets within a few $\mathrm{AU}$ of these stars. Less than $1 \%$ of $\mathrm{G}$ and $\mathrm{K}$ main sequence stars harbor brown dwarf companions with masses between 5 and $70 \mathrm{M}_{\mathrm{Jup}}$. Including Gliese $876 \mathrm{~b}, 8$ companions exhibit $m \sin i<5 M_{\text {Jup }}$ which constitute the best planet candidates to date. Apparently, $4 \%$ of stars have planetary companions within the range $m \sin i=0.5$ to $5 M_{\mathrm{Jup}}$. Planets are distinguished from brown dwarfs by the discontinuous jump in the mass function at $5 M_{\text {Jup }}$. About $2 / 3$ of the planets orbit within just $0.3 \mathrm{AU}$ due in part to their favorable detectability, but also possibly due to a real "pile up" of planets near the star. Inward orbital migration after formation may explain this, but the mechanism to stop the migration remains unclear. Five of eight planets have orbital eccentricities greater than that of our Jupiter, $e_{\mathrm{Jup}}=0.048$, and tidal circularization may explain most of the circular orbits. Thus, eccentric orbits are common and may arise from gravitational interactions with other planets, stars, or the protoplanetary disk. The planet-bearing stars are systematically metal-rich, as is the Sun, compared to the solar neighborhood.
\end{abstract}

\footnotetext{
${ }^{1}$ Astronomy Department, University of California, Berkeley, CA 94720
} 


\section{Introduction}

Velocity measurements of main sequence stars have revealed eight companions that have $m \sin i$ under $5 M_{\text {Jup }}$, including one companion presented here (Mayor et al. 1999, Marcy \& Butler 1998, Noyes et al. 1997, Cochran et al. 1997). Companions with $m \sin i$ less than $5 M_{\text {Jup }}$ are the best planet candidates to date (see section 2). These planets reside in circular and eccentric orbits. Their orbits are consistent with formation in dissipative circumstellar disks, followed by gravitational perturbations (cf. Lissauer 1995, Lin et al. 1996, Artymowicz 1997, Levison et al. 1998). The orbital semimajor axes are less than $2.5 \mathrm{AU}$, limited by the 10-year duration of Doppler surveys, with most orbits being smaller than $0.3 \mathrm{AU}$. This "piling-up" of planets near their host stars appears to be a real effect. Planets that orbit between 0.5 and $1.5 \mathrm{AU}$ would be easily detected with our current Doppler precision of $5 \mathrm{~ms}^{-1}$, but none has yet been found. Even a uniform distribution of orbital radii suggests that orbital migration in the protoplanetary disk may be an important process (Lin et al. 1996, Trilling et al. 1998).

The distribution of the $m \sin i$ of substellar companions reveals two populations. Our survey of $107 \mathrm{GK}$ dwarfs revealed none that had $m \sin i=10$ to 80 $M_{\text {Jup }}$ (Marcy \& Butler 1998). This non-detection of brown dwarfs shows that brown dwarf companions occur with a frequency less than $\sim 1 \%$, within $5 \mathrm{AU}$ of main sequence stars. Similarly, Mayor et al. $(1997,1999)$ surveyed $~ 550 \mathrm{GK}$ dwarfs, and found at most 4 companions with masses between 10 to $80 M_{\mathrm{Jup}}$. (Hipparcos astrometry has shown that six previously suspected brown dwarfs from that sample are actually H-burning stars.) These dynamical searches show incontrovertibly that brown dwarfs are rare within $5 \mathrm{AU}$ of dwarfs.

However, $\sim 5 \%$ of GK stars harbor companions with masses from 0.5 to 5

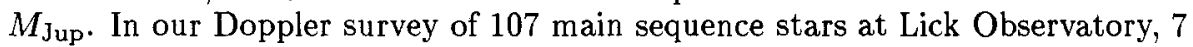
companions have $m \sin i=0.5$ to $5 M_{\mathrm{Jup}}$ (Marcy and Butler 1998, this paper). We present here that 7 th planet, orbiting the star Gliese 876 .

\section{Observations}

We measure Doppler shifts at both Lick and Keck Observatories by using the Hamilton and HIRES échelle spectrometers, (Vogt 1987, Vogt et al. 1994). We calibrate wavelengths and measure the spectrometer PSF for each exposure by using iodine absorption lines superimposed on the stellar spectrum (Butler et al. 1996). The resulting precision for bright stars $(V<6)$ is $3 \mathrm{~ms}^{-1}$ at Lick and $6 \mathrm{~ms}^{-1}$ at Keck, with the excess errors at Keck being due to a source that is not yet identified.

Representative Doppler measurements for stars that exhibit periods longer than 1 month are shown in Figure 1, which contains Lick data for $47 \mathrm{UMa}, 16$ Cygni B, $70 \mathrm{Vir}$, and $\rho \mathrm{CrB}$. Note that $70 \mathrm{Vir}$ does not meet the $5 M_{\text {Jup }}$ criterion as a planet, as it has $m \sin i=6.8 M_{\mathrm{Jup}}$. Indeed from our Lick survey of 107 GK dwarfs, that star exhibits the largest $m \sin i$, with all others showing $m \sin i$ $<4 M_{\text {Jup }}$.

Representative Doppler measurements for stars that exhibit periods less than 2 weeks are shown in Figure 2, plotted as a function of orbital phase. 
Velocity $(\mathrm{m} / \mathrm{s})$

Velocity $(\mathrm{m} / \mathrm{s})$

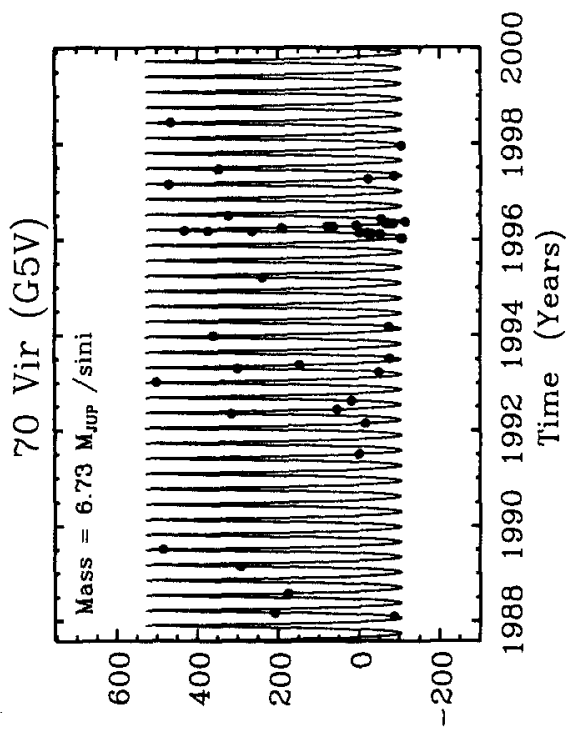

Velocity $(\mathrm{m} / \mathrm{s})$

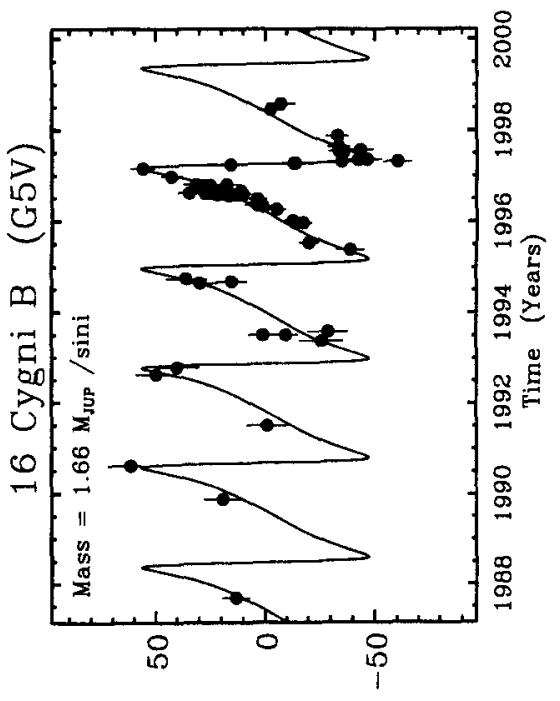

Velocity $(\mathrm{m} / \mathrm{s})$
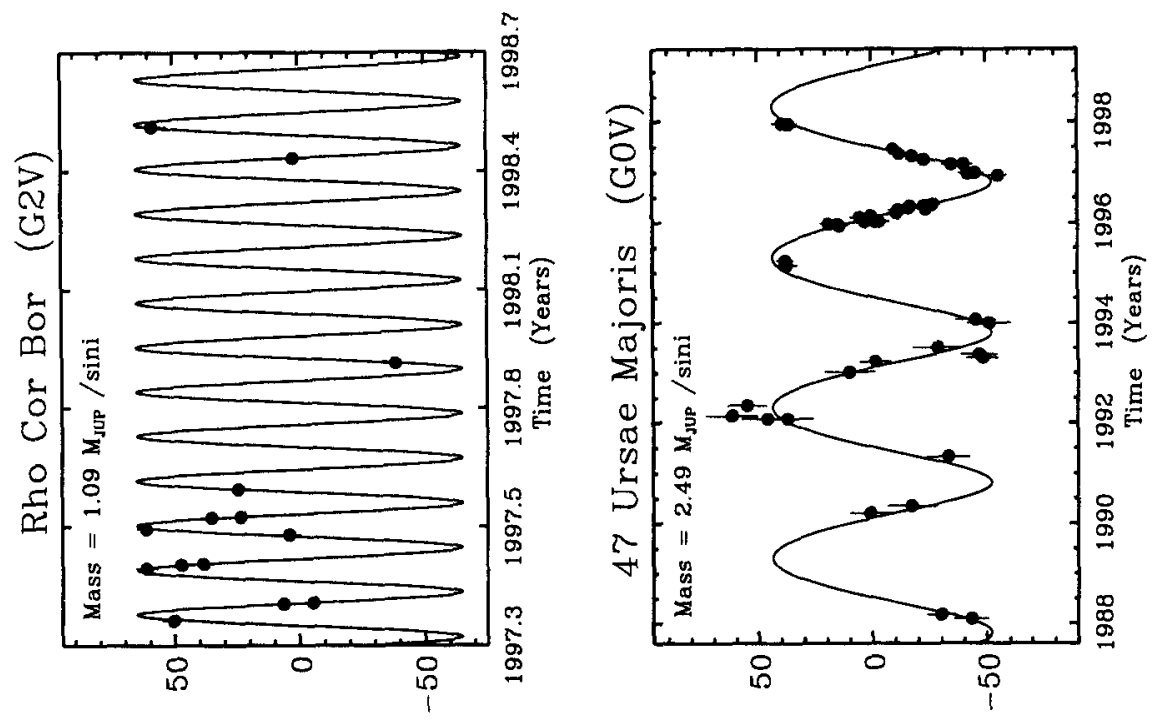

Figure 1. Doppler measurements for four stars having periods greater than 30 days, measured from Lick Observatory 

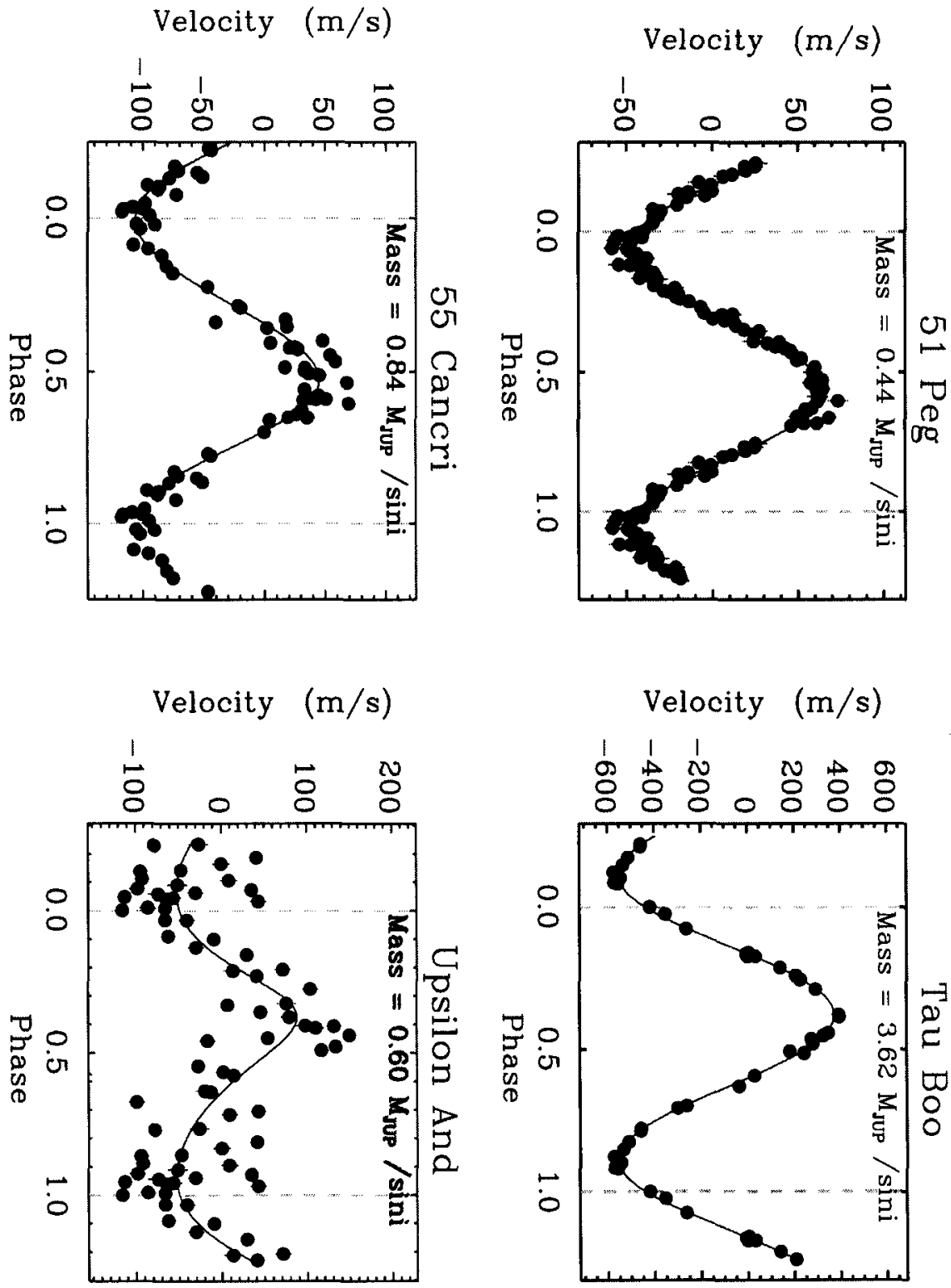

Figure 2. Doppler measurements for four stars having periods less than 15 days, measured from Lick Observatory 
This figure shows $51 \mathrm{Peg}, \tau$ Boo, Upsilon And, and $55 \rho^{1}$ Cnc. For the last two stars, the excess velocity residuals that are apparent to the eye appear to be correlated in time. This suggests the possibility that both of them contain additional companions having mass of order Jupiter-masses. To date, no secure Keplerian fits to any such further companions has been accomplished.

The orbital characteristics of all extrasolar planets known to date are listed in Table 1. The planetary orbits reveal a range of orbital characteristics. Orbital semimajor axes range from $0.05 \mathrm{AU}$ to $2.1 \mathrm{AU}$. Eccentricities range from 0.00 to 0.69 . From the Lick survey of 107 stars, the only companions found having $m \sin \ddot{i}$ in the substellar range $\left(0\right.$ to $\left.70 M_{\text {Jup }}\right)$ all have $m \sin i=0$ to $6.8 M_{\text {Jup }}$, with none having $m \sin i>7 M_{\mathrm{Jup}}$. This shows that "brown dwarfs" with mass above $7 M_{\text {Jup }}$ occur around less than $1 \%$ of GK dwarfs.

Table 1. Orbits of extrasolar planets having $m \sin i<5 M_{\text {Jup }}$

\begin{tabular}{llccrr}
\hline Star & $\begin{array}{l}\bar{P} \\
(\mathrm{~d})\end{array}$ & $\begin{array}{c}a \\
(\mathrm{AU})\end{array}$ & $\begin{array}{c}K \\
\left(\mathrm{~ms}^{-1}\right)\end{array}$ & $e$ & $\begin{array}{r}m \sin i \\
\left(M_{\text {Jup }}\right)\end{array}$ \\
\hline$\tau$ Boo & 3.3125 & 0.047 & 468. & 0.00 & 3.66 \\
51 Peg & 4.231 & 0.051 & 56.0 & 0.01 & 0.44 \\
$v$ And & 4.62 & 0.054 & 71.9 & $0.15:$ & 0.61 \\
$55 \rho^{1}$ Cnc & 14.65 & 0.11 & 75.9 & 0.04 & 0.85 \\
$\rho$ CrB & 39.6 & 0.23 & 67. & 0.11 & 1.1 \\
Gliese 876 & 61 & 0.21 & 217 & 0.27 & 2.1 \\
16 Cyg B & 799 & 1.6 & 50.3 & 0.687 & 1.67 \\
47 UMa & 1092 & 2.1 & 47.3 & 0.09 & 2.38 \\
\hline
\end{tabular}

The mass function in the substellar regime clearly rises rapidly at $5 M_{\text {Jup }}$ towards lower masses, as virtually all the companions found at Lick have $m \sin i<4$ $M_{\text {Jup. }}$. In figure 3 we show the histogram of $m$ sin $i$ that we obtained from the 11-year Doppler survey of 107 GKM stars from Lick. All of the companions have $m \sin i<7 M_{\text {Jup }}$, and none has higher $m \sin i$, up to $70 M_{\text {Jup }}$. This absence of companions above $m \sin i=7 M_{\mathrm{Jup}}$ is the most remarkable aspect of Figure 3 . It shows that there is not a continuum of the mass function from the H-burning limit at $75 M_{\text {Jup }}$ down to planetary masses. Instead, there is a dearth of brown dwarfs until the lowest masses around $5 M_{\mathrm{Jup}}$. At masses below $5 M_{\mathrm{Jup}}$, a new population of companions suddenly appears.

This rapid change in the mass function suggests that these companions of lowest mass require a new designation and probably formed in a qualitatively different way from the brown dwarfs. These companions below $5 M_{\text {Jup }}$ will be referred to as "planets". We do not know if these planets formed by the same set of processes that yielded those in our Solar System. Future observations and theoretical work will be required to make that association securely. We caution against defining planets by a set of pre-conceived characteristics such as circular and co-planar orbits, multiple companions, rocky cores, etc.). The diversity of physical processes that operate in protoplanetary disks may yield an unexpected diversity of planetary systems. 


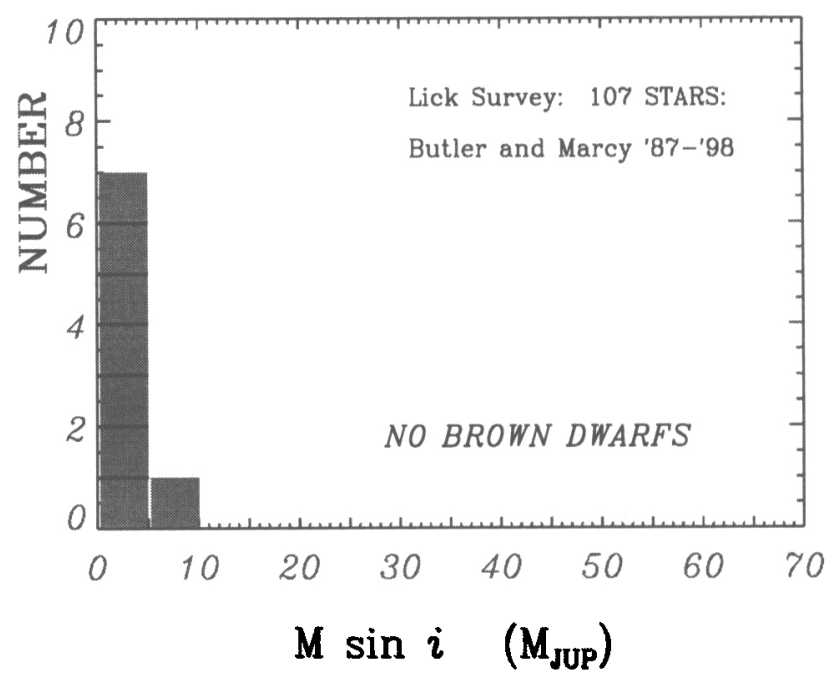

Figure 3. Histogram of $m \sin i$ for all companions discovered in the Doppler survey from Lick Observatory. All companions have $m \sin i$ $<7 M_{\text {Jup }}$. No companions have $m \sin i>7 M_{\text {Jup }}$, implying that brown dwarfs occur around less than $1 \%$ of main sequence stars. The companions having $m \sin i<5 M_{\mathrm{Jup}}$ are considered good candidate planets.

There is no selection effect that would tend to favor detection of the lowest mass companion $\left(0-10 M_{\mathrm{Jup}}\right)$. Instead, selection biases all favor detection of the more massive companions $\left(10-70 M_{\text {Jup }}\right)$ which produce the largest reflex velocity in the star. Remarkably, we found only low-mass substellar companions having $m \sin i<7 M_{\text {Jup }}$.

Of course, nature almost certainly manufactures some brown-dwarf companions between 10 and $70 M_{\text {Jup }}$ which orbit within $5 \mathrm{AU}$. The companions to $70 \mathrm{Vir}$ and HD 114762 may be such brown dwarfs (Marcy and Butler 1996, Latham et al. 1989), as may be 4 other companions having $m \sin i \sim 40 M_{\text {Jup }}$ (Mayor et al. 1999). However, "brown dwarf" companions are clearly rare compared to planets within $5 \mathrm{AU}$.

The range of orbital eccentricities, $e=0$ to 0.69 , exhibited by the extrasolar planets implies that some perturbative or scattering processes must occur after dissipation of the protoplanetary disk. If such scattering processes are common in young planetary systems, then our Solar System may represent the rare case in which circular orbits prevailed, perhaps by chance. Indeed the circular orbit of Jupiter itself permits a high density of smaller planets that would otherwise be rendered dynamically unstable were Jupiter in an eccentric orbit.

The closest 3 planets (51 Peg, $\tau$ Boo, and $v$ And) all have nearly circular orbits (and that of $55 \mathrm{CnC}$ is nearly circular too). These orbits may have been tidally circularized. The theory of tidal circularization remains uncertain due to challenges associated with treating the tidal dissipation. Nonetheless it appears that for $51 \mathrm{Peg}$ the tidal circularization time scale is $\tau_{\text {circ }} \approx 2 \times 10^{9} \mathrm{yr}$ (Rasio 


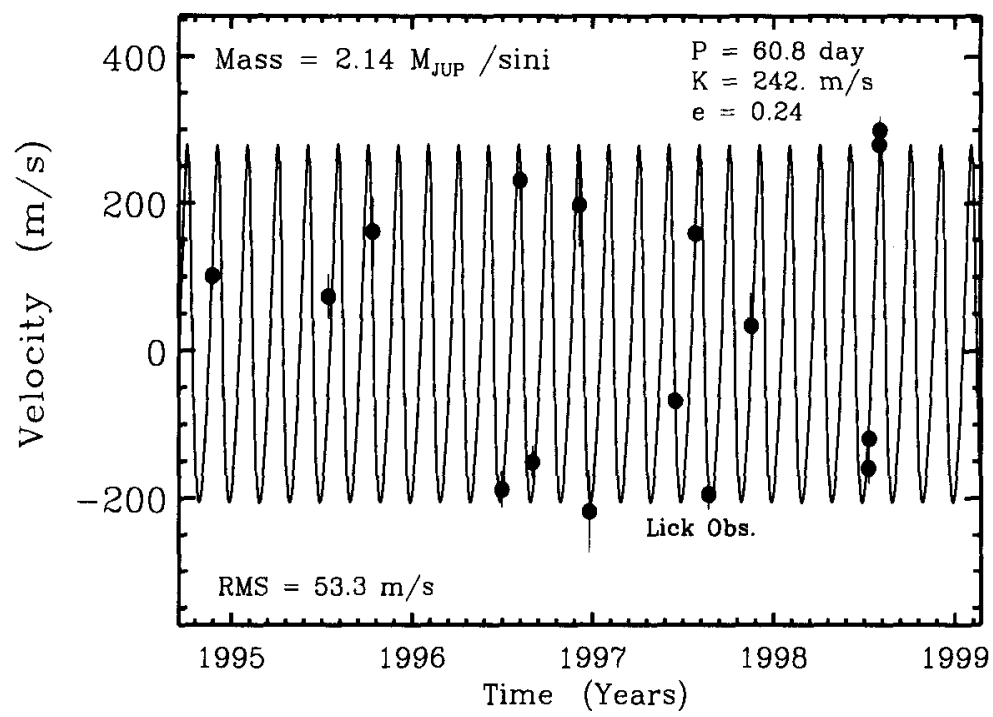

Figure 4. Doppler measurements for Gliese 876 from Lick Observatory. The Doppler variations were apparent by late 1996 .

et al. 1996, Marcy et al. 1997, Terquem et al. 1998). The planet around $55 \rho^{1}$ Cnc may be too distant to have been circularized as is the planet arourd $\rho$ CrB.

\section{A Planet Detected Around Gliese 876}

Gliese 876 has spectral type M4V and a parallax of 0.213 arcsec (Perryman et al. 1997). The mass of the star Gliese 876 can be estimated from its K-band apparent magnitude $(K=5.04)$ and parallax, to derive, $M_{*}=0.32 \pm 0.03 M_{\odot}$ (Henry \& McCarthy 1993). Gliese 876 is chromospherically inactive (Delfosse et al. 1998), implying that intrinsic velocity variations should be less than 10 $\mathrm{ms}^{-1}$ (Saar et al. 1998).

The first observations were made in 1994.9 (at Lick) and in 1997.4 (at Keck), and both data sets extend to the present. The Lick velocities are shown in Figure 4 and the Keck velocities are shown in Figure 5. In 1996, we noticed the large velocity variations of Gliese 876 in the Lick data. The star is so faint $(V=10.1)$ that we decided to augment the Lick velocities with Keck observations. By 1998 May we had demonstrated a mutually consistent Keplerian fit from both telescopes (Marcy et al. 1998). The combined Lick and Keck velocities are shown as a function of orbital phase in Figure 6 . The difference in the velocity zero-points from Lick and Keck was established by minimizing the RMS to the joint Keplerian fit.

The orbital period of Gliese 876 is $P=60.7 \pm 0.1 \mathrm{~d}$, and the eccentricity is $e=0.26 \pm 0.03$. The implied orbital semi-major axis is $0.21 \pm 0.01 \mathrm{AU}$, and $m \sin i=2.1 \pm 0.2 M_{\mathrm{Jup}}$. 


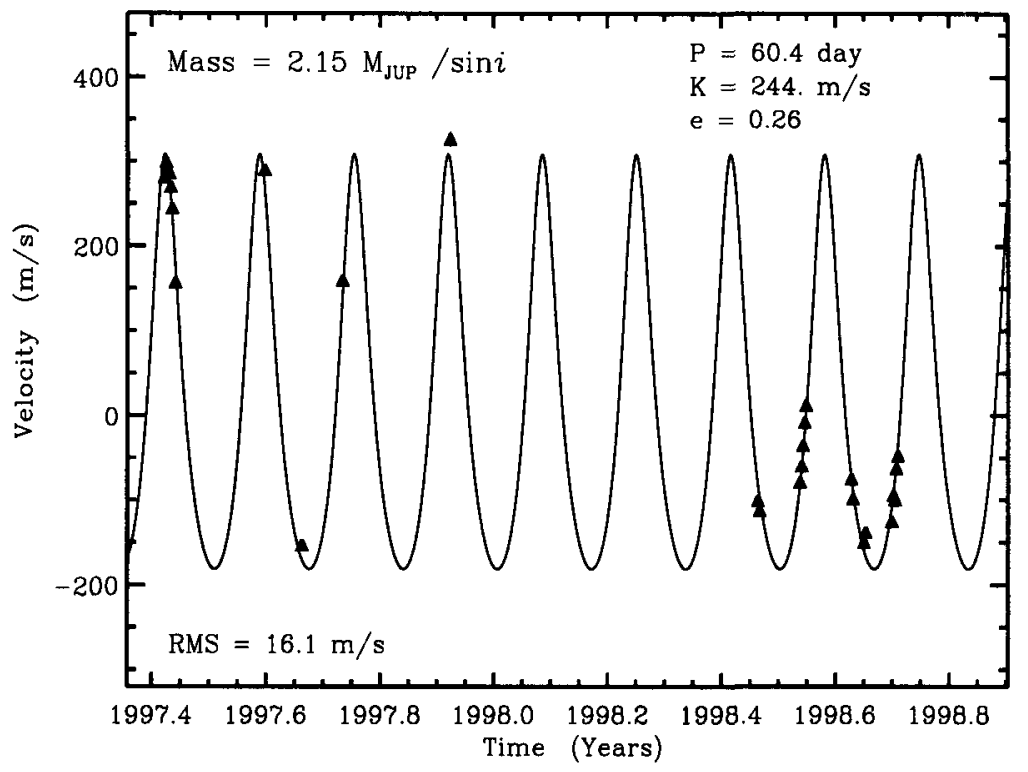

Figure 5. Doppler measurements for Gliese 876 from the Keck Observatory. The orbital parameters are consistent with those from Lick Obs.

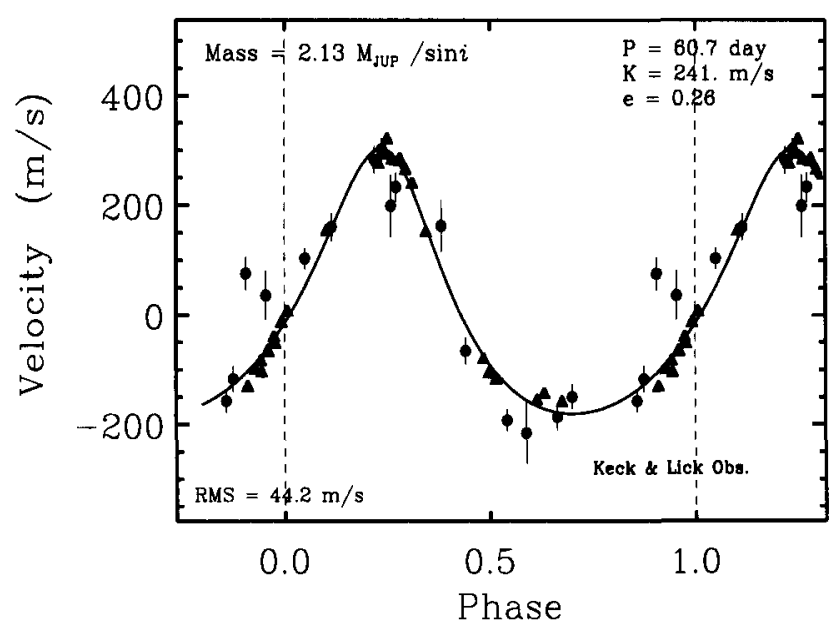

Figure 6. Doppler measurements from both Lick and Keck for Gliese 876 as a function of orbital phase. The two sets of velocities have a zero-point difference optimized for best Keplerian fit here. The two velocity sets agree and reveal a planet in an eccentric orbit that has $m \sin i=2.1 M_{\text {Jup }}$. 
Gliese $876 \mathrm{~b}$ appears to satisfy our criterion for planetary status as its $m \sin i$ is $2 M_{\text {Jup }}$, less than $5 M_{\text {Jup. }}$. It is the first planet found around an $M$ dwarf. At a distance of $4.7 \mathrm{pc}$, the planet may be detectable by future imaging or stellar astrometry, as the angular separation between star and planet is 0.044 arcsec. The astrometric angular semiamplitude of the star will be $0.29 / \sin i$ mas.

Acknowledgments. We are grateful to Tony Misch and Tom Bida for technical help at the telescope. We thank A. Cumming, W. Cochran, A. Hatzes, M. Mayor, D. Queloz, S. Udry, S. Vogt, G. Gonzalez, D. Lin and J. Lissauer for valuable discussions. Funding for this work came from NSF (AST 9520443 to GWM) and from NASA (NAGW 5125 to GWM).

\section{Discussion}

Cochran: I am not yet at the point of being worried about the relative timescales for planet formation, disk dissipation and viscous disk evolution. The disk is made up of gas, dust and planetesimals. The viscous evolution of the disk is primarily governed by the gas, which is difficult to measure directly. The IR observations of the disk lifetimes refer to the dust. The planetesimals can continue to evolve and form planets well after the gas and dust have been dispersed.

Mayor: In addition to your planet candidates, have you detected, in your survey, large amplitude r.m.s.?

Marcy: Yes, approximately a dozen of our 300 Keck stars exhibit a velocity r.m.s. between 100 and $1000 \mathrm{~ms}^{-1}$.

Noyes: Regarding your statement that the star $v$ And shows significant variation in the residuals to its 4.6-day orbit, with a characteristic timescale of several years, I would like to note that the AFOE, which has been monitoring this star since mid-1995, has also detected a systematic variation of the same residuals, with an amplitude of the order of $100 \mathrm{~ms}^{-1}$ and characteristic timescale of several years. It is too early, however, for us to conclude whether these variations represent the Keplerian orbit of a low-mass companion, or whether they are due to effects induced by magnetic activity - not unexpected given the spectral type (F8) and $v \sin i\left(11 \mathrm{kms}^{-1}\right)$ of the star. Of course, continuous monitoring over the next few years should resolve this important issue.

Tokovinin: The period distribution of close companions in multiple stars has something in common with that of planetary companions: there are many with periods from 3 to 5 days, then there is a partially filled gap, and companions are again more numerous for periods of more than a few years.

Vinko: Some of the 51 Peg-type stars seem to have very high metallicity. Do you have a comment on that?

Marcy: Yes, most of the planet-bearing stars are metal-rich, including the 51Peg members. Among the 8 stars that have $m \sin i<7 M_{\text {Jup }}, 7$ have a metallicity which is above the average of stars in the solar neighborhood. 


\section{References}

Artymowicz P. 1997. in Proceedings of the Workshop, Brown Dwarfs and Extrasolar Planets, held at Tenerife, Spain, March 1997, ed. R. Rebolo,E.L .Martín, M.R. Zapatero Osorio, 152-161.

Butler, R. P., Marcy, G. W., Williams, E., McCarthy, C., Dosanjh, P., \& Vogt, S. S. 1996, PASP, 108, 500.

Butler, R. P., Marcy, G. W., Williams, E., Hauser, H. \& Shirts, P. 1997, ApJ, $474, \mathrm{~L} 115$.

Cochran, W. D., Hatzes, A. P., Butler, R. P. \& Marcy, G. W. 1997, ApJ, 483, 457 .

Delfosse, X., Forveille, T., Perrier, C., Mayor, M. 1998, A\&A, 331, 581.

Latham, D., Mazeh, T., Stefanik, R. P., Mayor, M., Burki, G. 1989, Nature, 339,38

Levison, H. F., Lissauer, J. J., Duncan, M. J. 1998, AJ, in press.

Lin, D. N. C., Bodenheimer, P. \& Richardson, D. C. 1996, Nature, 380, 606.

Lin, D. N. C., Ida S. 1997. Ap. J. 477, 781

Lissauer, J. J. 1995. Icarus 114, 217

Marcy, G. W. \& Butler, R. P. 1998, ARAA, 36,57.

Mayor, M., Queloz, D., Udry, S. \& Halbwachs, J.-L. 1997, in Astronomical and biochemical origins and search for life in the Universe, IAU Coll. 161, ed. C.B.Cosmovici, S.Bowyer, and D.Werthimer, p313

Mayor, M., Queloz, D., Udry, S. \& Halbwachs, J.-L. 1999, To appear in Precise Radial Velocities, IAU Coll. 170, to appear in ASP Conference Series.

Noyes, R. W., Jha, S., Korzennik, S. G., Krockenberger, M., Nisenson, P., Brown, T. M., Kennelly, E. J., Horner, S. D. 1997, Ap.J.Lett. 483, L111

Perryman, M. C. et al. 1997, A\&A, 323, L49. The Hipparcos Catalog

Rasio, F. A. \& Ford, E. B. 1996. Science 274, 954

Terquem, C., Papaloizou, J. C. B., Nelson, R. P. \& Lin, D.N.C. 1998, ApJ, 502, 788

Trilling, D., Benz W., Guillot T., Lunine J. I., Hubbard W. B., Burrows A., 1998, Ap.J., 500, 428

Vogt, S. S. 1987, PASP, 99, 1214.

Vogt, S. S. et al. 1994, Proc. Soc. Photo-Opt. Instr. Eng., 2198, 362

Weidenschilling, S. J. \& Marzari, F. 1996. Nature 384, 619 